\title{
A cost-effective model for monitoring medicine use in Namibia: Outcomes and implications
}

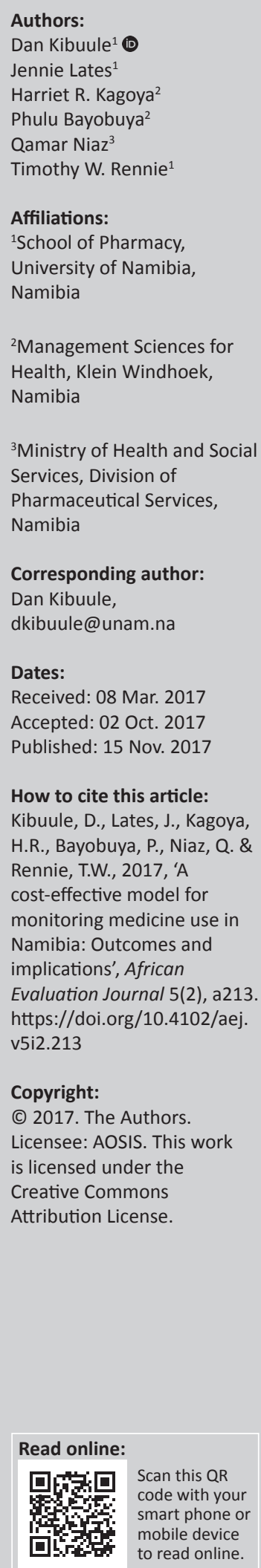

Background: Routine monitoring of medicine use is costly. Medicine use monitoring in most low- and middle-income countries is heavily reliant on donor support, which is not sustainable. Innovative models to close gaps in monitoring of medicine use are critical towards strengthening pharmaceutical services.

Objective: To pilot an inter-institutional collaborative model for monitoring medicine use in Namibia over a three-year period, 2013-2015.

Methods: An interventional analytical design that piloted an inter-institutional collaborative model for monitoring medicine use in public health facilities in Namibia was followed. Three key stakeholders - the Ministry of Health and Social Services (MoHSS) division of pharmaceutical services, University of Namibia School of Pharmacy and United States Agency for International Development-funded Systems for Improved Access to Pharmaceutical Services (SIAPS) project - collaboratively designed and implemented a concept model, tools and guidelines for routine medicine use assessment. The model integrated medicine use monitoring as a component of the annual rural placements of Bachelor of Pharmacy students at public hospitals. The pharmacists at the hospitals and MoHSS provided support and supervised the students prior to, during and after the placement. Each student undertook a mini-project on medicine use at the facilities which included data collection, analysis as well as reporting using the World Health Organization or International Network of Rational Use of Drugs indicators. These were subsequently aggregated by the university with technical assistance from SIAPS and findings reported to the Ministry. Data collected by the students on hospital placements were entered in Microsoft Excel ${ }^{\circledR}$ template for descriptive analysis for patient care indicators. All students discussed their findings with health facility supervisors.

Results: The collaborative efforts enhanced local institutional and students' capacity on analysing, reporting and presentation of data on medicine use. A total of three medicine use surveys (MUS) involving over 1938 patients were conducted from 2013 to 2015. The local capacity to conduct medicine use evaluation (MUE) was increased among 74 pharmacy students. At least 15 public hospitals in 12-14 regions participated in the MUS. Findings reveal $83 \%$ of prescribed medicines were dispensed; $53 \%-57 \%$ patients were satisfied with medicine information; $50 \%-59 \%$ of patients felt they waited too long (consultation time of more than $3 \mathrm{~h}$ ) before getting their medicines; over $80 \%$ patients did not know how to take their medicines correctly; $56 \%-80 \%$ of dispensed medicines were labelled correctly.

Conclusions: A multisectoral collaborative model is cost-effective in medicine surveys, if there are mutual benefits. Student placements provide an opportunity to build local capacity for routine MUE. Ministries of Health should utilise this innovative approach to assess service delivery.

\section{Introduction \\ Background}

Access to quality, safe and cost-effective medicines is a fundamental human right (Toebes 2001; WHO Medicine Strategy 2005). An efficient system for monitoring medicine use is a key element for successful health care delivery. Unfortunately, active monitoring of medicine use in most public health settings in lower- and middle-income countries (LMICs) is underfunded or mainly donor funded, which is not sustainable (Bigdeli et al. 2012). The inconsistent monitoring of medicine use in LMIC is mainly because of lack of budgetary commitment to this activity (Bigdeli et al. 2012). In most resource-constrained countries, monitoring of medicine use is performed on an ad hoc basis and often based on a passive self-reporting system. Self-reported data captured in the pharmaceutical management information system (PMIS) are rarely audited. This is mainly as a result of limited in-country capacity and capabilities among LMIC for active medicine use 
monitoring (Waako et al. 2009). In Namibia, systems to optimise existing capacity for medicine use monitoring among stakeholders is limited. The World Health Organization (WHO) records collaborative models to improve patient care and health care outcomes. However, this has not been developed in the LMIC (Gittell, Godfrey \& Thistlethwaite 2013). Collaborative efforts also reduce costs and improve the quality of health care services (Kilo 1998). An inter-institutional collaborative model (Figure 1) between three institutions Ministry of Health and Social Services (MoHSS), University of Namibia School of Pharmacy (UNAM-SoP) and Management Sciences for Health (MSH) - as development partners can optimise the limited budgets for M\&E on medicine use and improve outcomes. Unfortunately, countries and local institutions with capacity for M\&E on medicine use often work in silos with limited integration of activities (Massele et al. 2015). Irregular M\&E of medicine use may lead to lack of valid information to inform decisions on medicine use. This may lead to inappropriate use of medicines, wastage of medicines and resources and unachieved treatment outcomes. The WHO medicines use indicators in most LMIC remain suboptimal (WHO 2002). These include overuse of antibiotics, high number of medicines per prescription, overuse of injections and non-generic prescribing. The suboptimal medicine use indicators correlate with rise in antimicrobial resistance (AMR) and disease burden in the sub-Saharan African region. This consequently leads to use of medicines for longer treatment durations than may be required, thus increasing cost of health care. Timely information on patterns of medicine use informs treatment policies and improves outcomes (Bigdeli et al. 2012).

Currently Namibia uses a passive report of PMIS for reporting indicators on medicine use. This is implemented by the MoHSS and all public health facilities self-report on medicine use. The validation of the PMIS data from health facilities through active surveys on medicine use was last done in 2001 (Lates \& Shiyandja 2001). This self-report system may not be optimal and should be validated by an active surveillance to give additional information on the programmatic gaps at the individual facilities.

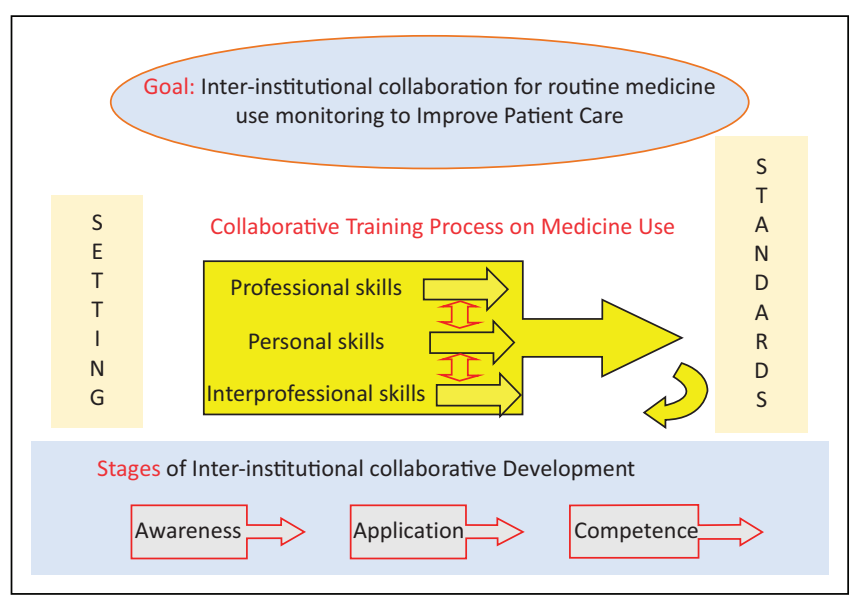

Source: Adapted from Charles, Bainbridge and Gilbert (2004)

FIGURE 1: Inter-institutional collaborative model for best practices on medicine use evaluation.

\section{Trends}

'Health Care Delivery models of the future clearly envision teams of health care providers working together to meet patient needs'. The inter-professional and inter-institutional collaborative efforts on monitoring of medicine in Namibia are limited by funding and models. The WHO has recommended collaborative efforts among institutions and professionals to optimise resources in monitoring medicine use and improve the quality of care $\mathrm{WHO}$ Medicine Strategy 2005). The WHO estimates that $50 \%$ of medicines are used inappropriately. This is highest in LMIC with limited resources. In Namibia, the PMIS indicates that medicine use indicators for antibiotic use and average of medicines per prescription do not meet national or global medicine use targets. These indicators have consistently remained above the WHO targets (Gbesemete \& Gerdtham 1992). Routinely, national medicine use evaluation (MUE) to validate PMIS indicators are limited with the last active survey in 2001. Consequently, there is a need for a system to optimise the meagre resources for active surveillance of medicine use indicators in Namibia.

\section{Problem statement}

\section{Key focus}

Expenditures on medicines rank highest on most health care budgets in LMICs (Stuart, Doshi \& Terza 2009). There are growing concerns that inappropriate medicine use and wastages increase costs of health care. The WHO estimates that over $50 \%$ of medicines in LMIC are used inappropriately (WHO 2002). This study focuses on piloting an innovative inter-institutional approach for sustainable monitoring of medicine use in public health care facilities in Namibia. Monitoring of medicine use is a critical element for successful delivery of pharmaceutical services and health care (Das et al. 2007). However, an efficient system to continuously monitor and evaluate medicine use requires a budgetary commitment of up to $10 \%$ of the total health care budget (Hardy, Phillips \& Lawrence 2003; Koenig et al. 2008). The last national survey on medicine use in Namibia was conducted in 2001, more than 16 years ago (Lates \& Shiyandja 2001). This is of major concern as data collected from the PMIS have not been validated. The infrequent MUE in LMICs such as Namibia underlines the problem of lack of capacity and capabilities for M\&E of medicine use. Systems for monitoring medicine use in the LMIC are heavily donor reliant - this is not sustainable in the wake of the global economic crunch (Bergman et al. 1980). In addition, self-reported indicators on medicine use captured by Health Management Information Systems (HMIS) are seldom audited. Infrequency in monitoring of medicine use may lead to adverse public health outcomes - including interrupting access to essential medicines or wastages (Stuart et al. 2009). Consequently, this study focuses on piloting a novel interinstitutional collaborative approach to routine and costeffective monitoring of medicine use to provide guidance on medicine use on an annual basis in Namibia.

\section{Objectives}

The main aim is to pilot an inter-institutional collaborative cost-effective model for active monitoring of medicine use in 
Namibia. The study also determined the medicine use patient care indicators and progress of the collaborative efforts among the institutions.

\section{Contribution}

This paper highlights a cost-effective model for monitoring medicine use among LMICs. Such models are necessary to optimise medicine use and treatment outcomes. This model will minimise budget wastages and provide routine information for decision-making regarding medicine use. These models suggest a mechanism to optimise local capacity and minimise over-reliance on donor funds for monitoring of public health care. The model also highlights better approaches to synergise efforts among local institutions for collaborative monitoring of medicine use.

\section{Literature review}

\section{Medicine use}

Wastage of medicines through inappropriate use particularly with antimicrobial antibiotics is a global public health problem (WHO 2002; Kunin 1993). Over 50\% of medicines are prescribed and dispensed irrationally (WHO 2002). The inappropriate use of medicines is highest in LMICs, particularly in the sub-Saharan Africa. Non-adherence to medication such as antiretroviral and antituberculosis medicines is another form of inappropriate use of medicines at the level of patient. This has had devastating effects on health care outcomes in these regions. Currently, the subSaharan region has the highest burden of infectious diseases including HIV, tuberculosis and malaria and there is a growing burden of AMR (Lopez et al. 2006). In Namibia the medicine use indicators in the public care are suboptimal particularly the prescribing and use of antibiotics (MoHSSNamibia 2015, Kibuule et al. 2017).

\section{Models for monitoring medicine use}

Inter-institutional and inter-professional best practice models for medicine use monitoring are ineffectively and inconsistently used in training institutions and practice settings (Kilo 1998). Two models are used to collect data on medicine use - these include active surveillance using MUEs and passive methods using electronic PMIS systems that aggregate data (Blumenthal \& Tavenner 2010; MoHSSNamibia 2015). The active surveillance is the gold standard as most health facilities in LMIC do not have the capacity to monitor medicine use (Waako 2009). Medicine surveys also validate data from the PMIS and provide a true picture of medicine use at the facilities. Unfortunately, active surveillance methods are expensive and rarely used in LMIC. This is depicted by the frequency of active surveillance of medicine use with the most recent national survey on medicine use being last done in 2001. This calls for an innovative system to routinely report medicine use indicators from both the PMIS and MUE. Reliance on electronic reporting systems carries errors of data entry and aggregation of data as well as challenges when there is a failure in the system or capacity to update the system. This is so at many health facilities where patient and work overload may be a barrier for completing electronic records (Waako et al. 2009).

\section{Gaps in medicine use monitoring}

Inter-institutional and inter-professional collaboration on medicine use is inconsistent. There is need for a best practice model for active medicine use monitoring in Namibia. There is limited capacity for routine MUE in Namibia. A high workload at health facilities is a major barrier to completion of electronic data entry and there is no system to ensure the quality and validate the accuracy of the data entered in the PMIS. The routine survey of medicine use in Namibia is infrequent and the need for a cost-effective approach is urgent.

\section{Research method and design Setting}

Data on medicine use were collected from health facilities of MoHSS, Namibia. These public health facilities were hospitals in all the 14 regions of Namibia. These included district hospitals and regional referral hospitals that are used as placement training sites for the Bachelor of Pharmacy students of the UNAM for the rural placement training. All the health facilities had a PMIS system in place for monitoring medicine use and the responsible person was a hospital pharmacist or regional pharmacist. The students are placed at the site for four weeks to undertake rural placement training that includes conducting a MUE and rational use of medicines. Regional pharmacists aggregate and report on medicine use indicators on a monthly or biannual basis to the MoHSS division of pharmaceutical services.

\section{Design}

The pilot project utilised an interventional design where an inter-institutional model for monitoring medicine use at public health facilities was implemented. In addition, MUEs were recommended by WHO (1993) to collect data on patient care indicators at public health facilities in Namibia. Data collection was done using quantitative methods using the tool shown in Appendix 1. Data on the four medicine use indicators - that is, proportion of medicines prescribed with generic names, proportion of prescriptions with an antibiotic, percentage of prescriptions with an injection and average number of medicines per prescription - were collected. A minimum of 30 medicine use encounters were assessed for each facility, each year of data collection. Data were collected by three cohorts (2013-2015) of second-year Bachelor of Pharmacy students on their rural placement rotation.

\section{Procedure}

A data collection tool was developed in collaboration with the UNAM-SoP, the MoHSS Pharmaceutical division and the MSH, Namibia. The tool was pretested on 10 patients at Katutura State Hospital, Windhoek, and standardised (Appendix 1). The students were trained on data collection using the tool and aspects of quality assurance prior to the 
placement. Three cohorts of students participated in the data collection as part of their rural placement training. Clearance to collect data at the health facilities was provided by the MoHSS. The study population was all patients receiving care at outpatient departments or units of the hospitals. Data were collected through patient exit interviews at the out-patient pharmacy. Each of second-year pharmacy students collected data from 30 patients, as part of training within the placement at a specific health facility using a standardised tool. Patients were randomly selected based on the out patient register. Prior to collection of data, patients were briefed on the study and participated in the study after providing a written informed consent. Only adult patients ( $>18$ years) who gave a written informed consent were included in the study. The data were checked for completeness by each student and entered into an Excel data collection tool.

\section{Analyses}

Quantitative data on 14 items entered from all the students were aggregated into one Excel data set. This aggregated data set was used as a research project for final-year B.Pharm. students under the supervision of representative researchers that is, MoHSS, UNAM and MSH. Each student performed basic data analysis on the data that they collected and presented results mainly in the form of percentages. The data were analysed using descriptive statistics - using means and absolute and relative frequencies in Excel. The main outcome variables were the $\mathrm{WHO}$ medicine use indicators and the level of satisfaction with dispensing services. The factors associated with the level of satisfaction and medicine use indicators were evaluated using bivariate analysis using chi-squared test and student's $t$-test. The findings were shared with the MoHSS Pharmaceutical division and presented to the hospital managers for reference in order to improve the use of medicines and strengthen the efforts to prevent AMR.

\section{Results}

\section{The inter-institutional collaborative model for monitoring medicine use}

The model was adapted from models for best practices for inter-professional collaboration (Charles, Bainbridge \& Gilbert 2004). The goal of inter-institutional collaboration was adapted for routine MUE to improve patient care in LMIC. The model requires identification of settings in which the MUE is to be done. The standards of performing the MUE in this setting are determined collaboratively by the team. The team agreed on items to be assessed and the methodologies to be used in the assessment. In this model the data collectors are medical students undertaking placement or internship programmes in these settings. The data collectors are collaboratively trained by the stakeholders on three skills related to professional and ethical skills to be maintained, the personal skills of data collection such as communication and inter-professional skills to encourage teamwork during the data collection process. In addition, the inter-institutional collaboration among the institutions should follow a development process with three stages including awareness stage, application stage and the competence stage. The awareness is the introduction of the concept of interinstitutional collaborative practice. At this stage, the roles of each institution and designate are identified and clarified and also require building inter-professional and inter-institutional relationships. The application stage is when advances in the depth and breadth of inter-institutional collaborations on medicine use evaluations are developed. This requires active team participation as well as interaction to apply their knowledge, skills and experiences on medicine evaluations. At this stage, competences on medicine use evaluations are developed including trust, communication and data collection and analysis. The last stage is the application of competences in the delivery of inter-institutional implementation of medicine use skills and knowledge as part of a team. The skills of each institution and designates are integrated into the actual practice of MUE. This requires active participation and decisions in collaborative MUEs.

\section{A strength weakness opportunities and threats analysis of the pilot of the inter-institutional collaborative model for medicine use}

Collaborative efforts between the three key institutions enhanced collaborative training on and active monitoring of MUE in Namibia. The WHO recommends IPE/IPC best practice models for transformative training that encourages building capacity by learning together in order to work together with the aim of providing quality health care and outcomes. The collaborative efforts between the three institutions improved trust as well as well as interprofessional and inter-institutional communication. The roles of the three institutions were clarified in the collaboration with UNAM helping with the research component, the MoHSS assisting with integration of learners into the practice sites and MSH-Namibia providing technical assistance in the development of tools and implementation of the MUEs. The collaboration also gave an opportunity for collecting patient level data on satisfaction with pharmaceutical services at the public health facilities nationwide. The team functioning between the three institutions has improved and similar collaborative efforts have been achieved with other programmes such as the Diploma in Pharmacy placements. This also gave an opportunity for the three institutions to take collaborative leadership on the various aspects of the MUE.

The collaborative efforts for medicine use required the following best practices: Clarity on the responsibilities of each institution. This requires the development of the plan and one of the institutions to coordinate the activities. The tertiary institutions are strategically placed to coordinate activities for training and research on medicine use. There was a need to agree on standards for inter-institutional collaboration on MUE so as to eliminate ambiguity, duplication and wastage of resources. The assessment also highlighted aspects of a healthy team and dynamics, that is, to have the right representatives of the different institutions to form the coordinating committee; these personnel should be committed 
and qualified to perform the duties with the right mix of competence in medicine use as well as empowerment and accountability to the activities. In addition, there is need for alignment of strategies for training and implementation of MUEs to the current needs and existing capacity as well as the level of practice of the student data collectors and setting in which the data is to be collected. The surveys strategically chose facilities that had a pharmacist to provide support supervision to the students. The collaboration also ensured that the training and surveys are executed within the same time period. The outcomes of the survey were similar for every student. Another important lesson is to use methods that are routinely used by the health facility to collect data and to not interfere in routine work at the facilities.

\section{Cost-effective model to optimise medicine use surveys}

\section{Outcomes of the medicine use surveys 2013-2015}

A total of four MUEs involving over 1938 patients have been conducted from 2013 to 2015 (Table 1). The local capacity to conduct MUE has been increased among 74 pharmacy students. At least 15 public hospitals in 14/14 regions have participated in the MUE. Findings from the available information on the medicine use indicator data collected in 2013-2015 were the following: 83\% of prescribed medicines were dispensed; $53 \%-57 \%$ patients were satisfied with medicine information; $50 \%-59 \%$ of patients felt they waited too long (consultation time of more than $3 \mathrm{~h}$ ) before getting their medicines; over $80 \%$ patients did not know how to take their medicines correctly (correct dose, duration, frequency); and $56 \%-80 \%$ of dispensed medicines were labelled correctly (Table 1). The reports are disseminated annually at the pharmacist forum.

\section{Ethical considerations, trustworthiness and validity}

Ethical considerations: The surveys were approved by the research and ethical review board of the MoHSS of Namibia. The study was approved by the UNAM-SoP Research Ethics Committee and the office of the permanent secretary MoHSS
(9-0/0001) as part of routine evaluation and training of students at health facilities (TT2013, TP2014, DP2015). The hospital managers also gave approval to collect data at the respective facilities. Written informed consent was obtained from all patients who participated in the study. No patient identifiers like names and patient numbers were collected. All information that was collected was kept confidential by use of codes to delink individual patients from the records.

Potential benefits and hazards: The study was not invasive and thus there was no serious risk to patients who participated in the interviews. The study had no financial benefits to the patients but rather offers opportunities to identify medicine use issues to inform health managers' decisions on improvements needed. Rational medicine use benefits all human race as it contributes to combating AMR, a global threat to humanity.

Recruitment procedures: Patients interviewed were randomly selected as they left the pharmacy area. Participation in the interview was voluntary and there were no consequences whatsoever of refusing to participate in the interviews.

Informed consent: Managers of the hospitals were informed of the data collection exercise and permitted data collection. Sampled patients exiting the pharmacy area were informed of the purpose of the interview and the benefits that would accrue from them providing relevant information. Only adult patients who expressed understanding of the information about the interviews and voluntarily consented to providing information were interviewed.

Data protection: Completed data collection forms were kept safe from damage, loss and unauthorised access. Students returned the completed data collection form to the assigned lecturers at UNAM-SoP, who maintained custody of the tools for subsequent reference and additional analysis.

TABLE 1: Medicine use indicators at health facilities in Namibia.

\begin{tabular}{|c|c|c|c|c|}
\hline Covariate & $\begin{array}{l}\text { Cohort } 1 \text { (2013) } \\
\text { Thandiwe Trish }\end{array}$ & $\begin{array}{c}\text { Cohort } 2 \text { (2014) } \\
\text { Pelekekae T }\end{array}$ & $\begin{array}{c}\text { Cohort } 3 \text { (2015) } \\
\text { Pucuta D }\end{array}$ & Total/mean \\
\hline Region coverage & 6 & 9 & 11 & 12 \\
\hline Facility coverage & 7 & 15 & 12 & 15 \\
\hline Respondents & 289 & 990 & 659 & 1938 \\
\hline Namibian nationals & - & $83 \%$ & $82 \%$ & $82.5 \%$ \\
\hline Mean age (years) & 33 & 44 & 39.5 & 38.8 \\
\hline Students trained & 14 & 33 & 27 & 74 \\
\hline Dispensed by pharmacist & $17.8 \%$ & $44.1 \%$ & $20 \%$ & 27.3 \\
\hline Sex (female) & $56.7 \%$ & $59.1 \%$ & $59.8 \%$ & 58.5 \\
\hline Satisfaction & $73 \%$ & $85.8 \%$ & $83.9 \%$ & $80.9 \%$ \\
\hline Medicines received as prescribed & - & $83.4 \%$ & $72 \%$ & $77.7 \%$ \\
\hline Know how to take medicines correctly & - & - & $89.7 \%$ & - \\
\hline Medicines adequately labelled & 56.4 & $80.1 \%$ & $41.4 \%$ & 59.3 \\
\hline Satisfied with information on medicines & $58.2 \%$ & $56.6 \%$ & $85.6 \%$ & $66.8 \%$ \\
\hline Overall quality indicator & $62 \%$ & $78.6 \%$ & 84.2 & $74.9 \%$ \\
\hline Average waiting time (hours) & - & - & 3.06 & 3.06 \\
\hline
\end{tabular}

Source: Authors' own work

The students Thandiwe Trish, Pelekekae T and Pucuta D were the university students who compiled the reports for each survey as part of their research projects. 
Trustworthiness: The findings are based on the discovery of patients' individual experiences of seeking and obtaining health and pharmaceutical services at the MoHSS hospitals on the days of the interviews.

Validity: The study tool was pretested among 10 patients at Katutura Sstate Hospital in Windhoek. This tool was adapted from the WHO/International Network of Rational Use of Drugs MUE tools (WHO 1998). The data collectors were inducted on the use of the tools and ensuring quality of data. The patients who participated in the study were randomly selected from the patient attendance registers. The methods for data collection are similar to MUE that was conducted by Lates and Shiyanga (2001).

\section{Discussion}

The main objective of the assessment was to develop a costeffective model for medicine use evaluation in Namibia. The study aimed at describing findings of an inter-institutional collaborative model and performed a strength weakness opportunities and threats analysis of the model.

\section{Medicine use evaluations}

The study aimed at providing a mechanism for consistent monitoring of medicine use in LMIC. To date, three consecutive MUE have been conducted in up to 12 regions of Namibia. This gives a national coverage of $86 \%$ for the medicine use evaluations in the past three years (2013-2015). Over the three years (2013-2015), the study found that patient care and dispensing indicators were suboptimal and vary by year and health facility. Various surveys among LMIC have reported suboptimal indicators of medicine use (Bergman et al. 1980; Lates \& Shiyandja 2001; WHO 2004). The collaborative efforts enhanced the capacity among institutions and pharmacy students to conduct and report on MUEs at facility level. This is in line with the WHO's strategy to promote collaborative efforts to improve health care and build capacity particularly in resource-limited settings (Kilo 1998). The satisfaction on pharmaceutical services is also not optimal and varies with health facilities. There is also variation in the waiting time at the health facilities and this affects the confidence in the care and influences health-seeking behaviours among the community. Some medicines are dispensed by non-qualified personnel and just over $50 \%$ of the patients know how to take the right doses of their medicines. Previous efforts have indicated the lack of human resources as a major predictor of irrational use of medicines (Waako et al. 2009)

\section{Model for best practices for medicine use evaluation}

A framework to enhance collaborative MUEs was developed. The collaborative model between a local university placement programme, the MoHSS public health facilities and development partners is cost-effective. The collaboration has yielded annual reports on the quality of dispensing services at health facilities in Namibia. The WHO recommends collaborative efforts between professionals to improve patient care (WHO 2002). Unfortunately, the inter-professional collaborations have only been applied during training and practice among health professionals. The collaborative efforts to ensure quality and consistent reporting on medicine use are inconsistent and ineffective in most countries. There are limited successful models for inter-professional collaboration on MUEs that optimise costs and provide a mechanism for routine reporting on the quality of services and patient care indicators (Hardy et al. 2003; Kibuule et al. 2017; Kilo 1998). This study provides a model and framework for training and development of inter-institutional collaboration during evaluation of medicine use on a routine basis. In this model, the key stakeholders pooled efforts to develop a tool for routine assessment of patient care indicators using existing systems at the public health facilities and programmes at the university.

\section{Strength weakness opportunities and threats analysis of the inter-institutional model}

The study aimed to identify the benefits and challenges of the inter-institutional collaborative model to reduce costs for medicine use evaluations. The main strength of the programme was strengthening the collaboration between stakeholders with minimal costs and optimising the competences. The assessment also developed teamwork among the institutions, designates and the students. There were opportunities to use existing placement programmes of the university at the health facilities to conduct routine MUEs at no costs. This in turn provided a service to the MoHSS to provide routine data on patient care indicators at the health facilities. The main weakness of the survey was that coverage of the facilities was restricted to facilities where students were placed and this excluded primary health care facilities. The main threat to the model is lack of commitment by the stakeholders for continuous improvement of the medicine use evaluations.

\section{Practical implications}

The study depicts a model to enhance inter-institutional collaborative efforts to minimise costs and improve the quality and frequency for conducting regular MUEs. Secondly, the study highlights a model framework for institutional collaborations on medicine use evaluations. The survey highlights standards for conducting the MUEs that have been developed to provide guidance to LMIC (Kibuule et al. 2015). A concept and tool to guide medical and pharmacy students to support the medicine use monitoring has been developed and implemented within the rural placements of the School of Pharmacy of the Ministry of Health. Other countries may take lessons from this model to forge collaborative efforts for MUEs to improve service delivery.

\section{Limitations of the assessments}

The main limitations of this survey were seeking ethical approval to conduct routine surveys on behalf of the Ministry of Health. This caused delays in collecting data in 
some facilities. There is need of integration of the MUEs in the strategic and operational plans of the various institutions involved.

\section{Recommendations}

The study recommends that inter-institutional and interprofessional collaborative efforts should be promoted by the MoHSS to enhance service delivery outcomes. The medical and pharmacy schools have a great potential of supporting medicine use evaluation. Enhancement of pre-service capacity for medicine use evaluations will improve future health care and reporting practices as well as close the gaps of human resource capacity in specific technical gaps. The concept and guidelines developed by the collaborative efforts in Namibia may be adapted by other LMIC to improve medicine surveillance efforts. There is need for in-country policy framework to guide inter-institutional collaborative efforts particularly when many parties are involved such as students.

\section{Conclusion}

The inter-institutional collaborative model between the UNAMSoP, the Pharmaceutical Division of the Ministry of Health and Management Sciences for Health greatly improved monitoring of medicine use in Namibia over the past four years. Although medicine use indicators remain suboptimal, collaborative efforts and teamwork remain the key components for improving medical and pharmaceutical service delivery.

\section{Acknowledgements}

The authors would like to acknowledge the permission from the Ministry of Health and Social Services to access the public health facilities for placements of students and to conduct the survey. The authors also appreciate the support given by the individual students to collect data during the placement as well as the pharmacists who worked as site supervisors during this placement. Special acknowledgement goes to Ms Toga Trish, Ms Pelekekae Tumo and Ms. Herondina Pucuta who compiled the three reports whose results have been included in this manuscript. Appreciation also goes to the US Agency for International Development, Systems for Improved Access to Pharmaceutical Services Program, for funding the Management Sciences for Health staff time for the technical assistance rendered in these studies. Technical support was provided by the Management Sciences of Health, Namibia.

\section{Competing interests}

The authors declare that they have no financial or personal relationships that may have inappropriately influenced them in writing this paper.

\section{Authors' contributions}

D.K. played a major role in conceptualising and implementing the studies, supervised implementation of the studies, data analysis and report writing. He performed additional data analysis and co-authored this manuscript. J.L. contributed to documentation of the study concept, supervised data collection and reviewed the manuscript. H.R.K. contributed to the annual study concept, developed initial data collection tools, implementation of the study and wrote the manuscript. B.P. gave technical insights on medicine use assessments, data collection, and reviewed the manuscript. Q.N. contributed to conceptualisation of the study, coordinated data collection in public health facilities and reviewed the manuscript. T.R. contributed to the study design, implementation and writing of the manuscript.

\section{References}

Bergman, U., Christenson, I., Jansson, B. \& Wiholm, B., 1980, 'Auditing hospital drug utilisation by means of defined daily doses per bed-day a methodological study', European Journal of Clinical Pharmacology 17(3), 183-187. https://doi. org/10.1007/BF00561898

Bigdeli, M., Jacobs, B., Tomson, G., Laing, R. \& Ghaffar, A., 2013, 'Access to medicines from a health system perspective', Health Policy and Planning 28(7), 692-704. https://doi.org/10.1093/heapol/czs108

Blumenthal, D. \& Tavenner, M., 2010, 'The "meaningful use" regulation for electronic health records', The New England Journal of Medicine 363(6), 501-504. https:// doi.org/10.1056/NEJMp1006114

Charles, G., Bainbridge, L. \& Gilbert, J., 2010, 'The University of British Columbia model of interprofessional education', Journal of Interprofessional Care 24(1), 9-18.

Das, R., Biswas, K., Panda, P., Khan, M.E. \& Homan, R., 2007, Strengthening financial sustainability through integration of voluntary counseling and testing services with other reproductive health services, Population Council, Frontiers in Reproductive Health, New York.

Gbesemete, K. \& Gerdtham, U., 1992, 'Determinants of health care expenditure in Africa: A cross-sectional study', World Development 20, 303-308.

Gittell, J., Godfrey, M. \& Thistlethwaite, J., 2013, Interprofessional collaborative practice and relational coordination: Improving healthcare through relationships, World Health Organization, Geneva.

Hardy, C., Phillips, N. \& Lawrence, T., 2003, 'Resources, knowledge and influence: The organizational effects of interorganizational collaboration', Journal of Management Studies 4(2), 321-347. https://doi.org/10.1111/1467-6486.00342

Kibuule, D., Lates, J., Niaz, Q., Mazibuko, G., Kagoya, H. \& Rennie, T., 2015, Concept and guidelines for medicine use assessment during students' rural placements, viewed 3 December 2016, http://apps.who.int/medicinedocs/documents/ s22148en/s22148en.pdf

Kibuule, D., Mubita, M., Naikaku, E., Kalemeera, F., Godman, B. \& Sagwa, E., 2017, 'An analysis of policies for cotrimoxazole, amoxicillin and azithromycin use in Namibia's public sector: Findings and therapeutic implications', International Journal of Clinical Practice, 71(2), 1-10.

Kilo, C., 1998, 'A framework for collaborative improvement: Lessons from the Institute for Healthcare Improvement's Breakthrough Series', Quality Management in Healthcare 6(4), 1-4. https://doi.org/10.1097/00019514-199806040-00001

Koenig, S., Riviere, C., Leger, P., Severe, P. \& Atwood, S., 2008, 'The cost of antiretrovira therapy in Haiti', Cost Effectiveness and Resource Allocation 14(6), 3. https://doi. org/10.1186/1478-7547-6-3

Kunin, C., 1993, 'Resistance to antimicrobial drugs - A worldwide calamity', Annals of Internal Medicine 118(7), 557-561. https://doi.org/10.7326/0003-4819-118-7199304010-00011

Lates, J. \& Shiyandja, N., 2001, Third National Survey on the use of drugs in Namibia's public health institutions, including monitoring the implementation of the National Drug Policy, Ministry of Health and Social Services, Windhoek, Namibia.

Lopez, A., Mathers, C., Ezzati, M. \& Jamison, D., 2006, 'Global and regional burden of disease and risk factors, 2001: Systematic analysis of population health data', The Lancet 367(9524), 1747-1757. https://doi.org/10.1016/S0140-6736(06)68770-9

Massele, A, Burger, J, Katende-Kyenda, NL, Kalemeera, F, Kenaope T, Kibuule D et al. 2015, 'Outcome of the first Medicines Utilization Research in Africa group meeting to promote sustainable and rational medicine use in Africa', Expert Review Pharmacoeconomics Outcomes Research 15(6),885-8.

MoHSS-Namibia, 2015, National Pharmacy Management Information Systems (PMIS) feedback report Apr-Sep 2015, Ministry of Health and Social Services Namibia, Windhoek.

Stuart, B., Doshi, J. \& Terza, J., 2009, 'Assessing the impact of drug use on hospital costs', Health Services Research 44(1), 128-144. https://doi.org/10.1111/j.1475 6773.2008.00897.x

Toebes, B, 2001, 'The right to health as a human right in international law', Refugee Survey Quarterly 20(3).

Waako PJ, Odoi-adome R, Obua C, Owino E, Tumwikirize W, Ogwal-Okeng J. et al 2009, 'Existing capacity to manage pharmaceuticals and related commodities in East Africa: An assessment with specific reference to antiretroviral therapy', Human Resources for Health 7(21), 1478-4491. https://doi.org/10.1186/14784491-7-21 
World Health Organization (WHO), 1993, How to investigate drug use in health facilities: Selected drug use indicators, WHO, Geneva.

World Health Organization (WHO), 2002, Promoting rational use of medicines: Core components, World Health Organization, Geneva.
World Health Organization (WHO), 2004, The world medicines situation, World Health Organization, Geneva.

World Health Organisation Medicine Strategy, 2005, Countries at the core, World Health Organization, Geneva. 


\section{APPENDIX 1}

\section{Data Collection Sheet for Calculation of Rural Attachment indicators}

\begin{tabular}{|c|c|c|c|c|c|c|c|c|c|c|c|c|c|}
\hline \multicolumn{6}{|c|}{$\begin{array}{l}\text { Data Collection Sheet for Calculation of Rural Attachment } \\
\text { indicators }\end{array}$} & \multicolumn{3}{|c|}{ HEALTH FACILITY: } & & & & & DATE: \\
\hline \multicolumn{2}{|c|}{ Question 1 (Q1): } & \multicolumn{12}{|c|}{ How many of the medicines prescribed did the patient receive? (write Number Dispensed (\# Dx) and Number Prescribed (\# Rx) } \\
\hline \multicolumn{2}{|c|}{ Question 2 (Q2): } & \multicolumn{12}{|c|}{ Is the patient satisfied with the information received with dispensing of their medication? } \\
\hline \multicolumn{2}{|c|}{ Question 3 (Q3): } & \multicolumn{12}{|c|}{ Does the patient know how to take ALL of their medicines correctly? } \\
\hline \multicolumn{2}{|c|}{ Question 4 (Q4): } & \multicolumn{12}{|c|}{ Does the label on ALL dispensed medicines contain all the information required for good dispensing practice? } \\
\hline \multicolumn{2}{|c|}{ Question 5 (Q5): } & \multicolumn{12}{|c|}{ Does the prescription include an antibiotic? } \\
\hline \multirow{3}{*}{$\begin{array}{l}\text { Case } \\
\text { No. }\end{array}$} & \multirow{3}{*}{\begin{tabular}{|c|} 
Column 1 \\
$\begin{array}{c}\text { Time of } \\
\text { interview }\end{array}$
\end{tabular}} & \multicolumn{2}{|c|}{ Column 2} & \multirow{3}{*}{$\begin{array}{c}\text { Column } 3 \\
\text { Sex (1=Male } \\
0=\text { Female) } \\
\end{array}$} & \multirow{3}{*}{$\begin{array}{c}\text { Col } 4 \\
\text { Age } \\
\text { (Years) } \\
\end{array}$} & \multirow{3}{*}{\begin{tabular}{|c|} 
Column 5 \\
Dispenser \\
P/A, P or Other \\
\end{tabular}} & \multirow{3}{*}{\begin{tabular}{|c|} 
Column 6 \\
Arrival Time \\
at HF \\
\end{tabular}} & \multirow{2}{*}{\multicolumn{2}{|c|}{$\begin{array}{c}\text { Column } 7 \\
\text { Answer Q1 }\end{array}$}} & \multirow{3}{*}{$\begin{array}{c}\text { Column } 8 \\
\text { Answer Q2 } \\
\text { 1=yes; } 0=\text { no } \\
\end{array}$} & \multirow{3}{*}{$\begin{array}{c}\text { Column } 9 \\
\text { Answer Q3 } \\
\text { 1=yes; 0=no } \\
\end{array}$} & Column 10 & Column 11 \\
\hline & & Has ID? & Namibian? & & & & & & & & & Answer Q4 & Answer Q5 \\
\hline & & (a) $1=y \in$ & es; $0=$ no (b) & & & & & (a) \# Dx & \# Rx (b) & & & $1=y e s ; 0=$ no & $1=$ yes; $0=$ no \\
\hline 1 & & & & & & & & & & & & & \\
\hline 2 & & & & & & & & & & & & & \\
\hline 3 & & & & & & & & & & & & & \\
\hline 4 & & & & & & & & & & & & & \\
\hline 5 & & & & & & & & & & & & & \\
\hline 6 & & & & & & & & & & & & & \\
\hline 7 & & & & & & & & & & & & & \\
\hline 8 & & & & & & & & & & & & & \\
\hline 9 & & & & & & & & & & & & & \\
\hline 10 & & & & & & & & & & & & & \\
\hline 11 & & & & & & & & & & & & & \\
\hline 12 & & & & & & & & & & & & & \\
\hline 13 & & & & & & & & & & & & & \\
\hline 14 & & & & & & & & & & & & & \\
\hline 15 & & & & & & & & & & & & & \\
\hline 16 & & & & & & & & & & & & & \\
\hline 17 & & & & & & & & & & & & & \\
\hline 18 & & & & & & & & & & & & & \\
\hline 19 & & & & & & & & & & & & & \\
\hline 20 & & & & & & & & & & & & & \\
\hline 21 & & & & & & & & & & & & & \\
\hline 22 & & & & & & & & & & & & & \\
\hline 23 & & & & & & & & & & & & & \\
\hline 24 & & & & & & & & & & & & & \\
\hline 25 & & & & & & & & & & & & & \\
\hline 26 & & & & & & & & & & & & & \\
\hline 27 & & & & & & & & & & & & & \\
\hline 28 & & & & & & & & & & & & & \\
\hline 29 & & & & & & & & & & & & & \\
\hline 30 & & & & & & & & & & & & & \\
\hline & & (A) & (B) & (C) & (D) & & & $(E)$ & (F) & (G) & (H) & (I) & (J) \\
\hline
\end{tabular}

